\title{
Le transport des gammagraphes
}

\author{
A. LAUMOND ${ }^{1}$
}

RÉSUMÉ Au 31 décembre 2008, les transports par route des gammagraphes seront réalisés selon toutes les prescriptions de la classe 7 de la réglementation des transports des matières dangereuses par route (A.D.R.). En effet les dispositions particulières de l'article 28 de "l'arrêté ADR modifié» du $1^{\text {er }}$ juin 2001, qui leurs étaient réservées ne seront plus applicables. Ce document présente les contrôles radiologiques à réaliser sur le gammagraphe et le véhicule et souligne les règles pour l'étiquetage le marquage et la signalisation, ainsi que les documents qu'il est nécessaire de trouver à bord du véhicule. Il aborde les exigences qui permettent une intervention efficace en cas d'accident de transport.

ABSTRACT Gammagraphes ${ }^{\circledR}$ transportation.

From December 31st 2008, all the Non Destructive Testing Gamma-Cameras (Gammagraphes ${ }^{\circledR}$ ) will be transported in accordance with the Dangerous Goods European transport rules, class 7. Indeed, specific measures of article 28 which were applicable to Gammagraphe won't be applicable. This document presents the radiological controls to be realized, and the labels to be used, and the documents needed for the administrative file. It approaches the requirements which allow an effective intervention in case of transport accident.

Keywords: Gammagraphs / transport / radiological controls / transport documents / accident of transport

\section{La réglementation des transports des matières radioactives par route, l'ADR, s'applique aussi aux gammagraphes}

Les transports des « matières radioactives » font l'objet de la classe 7 de l'A.D.R (Accord européen pour le transport des matières Dangereuses par Route) qui reprend totalement les recommandations du règlement ST-R-1 de l'Agence internationale pour l'énergie atomique (AIEA).

L'ADR constitue les annexes A et B, de l'arrêté français du $1^{\text {er }}$ juin 2001 « modifié » dit « arrêté ADR ». L'arrêté ADR a pour objet de compléter les dispositions de l'accord européen pour le territoire national.

Les dispositions de l'arrêté ADR spécifique au transport des gammagraphes sont supprimées depuis le 28 janvier 2008, mais ses dispositions restent en vigueur jusqu'au 31 décembre 2008 ( $c f$. article 49 de l'arrêté ADR).

1 EDF DPN, Unité Technique Opérationnelle, 6 avenue Montaigne, 93160 Noisy-le-grand, France. 


\section{La sûreté des transports des matières radioactives}

La probabilité qu'un colis de transport de matières radioactives soit impliqué dans un accident n'étant jamais nulle, la sûreté du transport consiste à éviter que dans la majorité des cas, de tels accidents puissent avoir des conséquences dommageables du point de vue radiologique. Cela nécessite que les colis assurent des performances de confinement et de protection adaptées aux risques engendrés par les matières transportées.

Les principes de sûreté appliqués pour réduire les risques d'impact radiologique de l'activité transports sont dans une logique de défense en profondeur de trois ordres :

1. Des performances en termes de protection sont imposées aux colis dès la conception.

2. Des précautions sont à prendre lors du transport.

3. Les interventions pour limiter les conséquences en cas d'accident doivent être préparées.

Au regard de la grande variété des colis transportés l'AIEA a défini une typologie des colis fondée sur les risques engendrés par le contenu transporté (et donc son activité). En fonction de son type (colis Excepté, du Type Industriel, du Type A, du Type B, du Type C), les prescriptions auxquelles doit correspondre le colis sont croissantes : les critères de performance de résistance des colis sont adaptés aux dangers potentiels de la matière transportée.

Par conception, le colis doit garantir le confinement de la matière, assurer la protection contre les rayonnements émis par la matière et prévenir tout risque de criticité lorsqu'il contient des matières fissiles, dans des situations de routine, d'incident et d'accident, prédéterminées couvrant la quasi-totalité des situations de transport réelles.

Ces conditions variées sont reproduites par des épreuves normalisées.

Ces règles visent à limiter à un niveau acceptable les nuisances, pour les personnels assurant le transport, le public et l'environnement.

Les gammagraphes sont pour la plupart des colis du Type $\mathrm{B}(\mathrm{U})$, donc les modèles de colis sont soumis à l'agrément de l'autorité de sûreté nucléaire (ASN). Il existe également des gammagraphes du Type A (colis du Type $\mathrm{B}(\mathrm{U})$ déclassés en Type A car l'activité contenue est $<\mathrm{A} 1$ ) : les modèles de ces colis ne sont pas soumis à l'agrément de l'ASN mais doivent présenter un certificat de conformité au modèle de colis du type A émis par le concepteur ou le propriétaire du colis. Pour démontrer la conformité à l'ADR du modèle de colis un dossier de sûreté doit être rédigé par son propriétaire. 
Le modèle de la source scellée (matière radioactive sous forme spéciale) contenue dans le gammagraphe est dans tous les cas soumis à l'agrément de l'autorité compétente.

\section{Quelques définitions}

Colis du Type A : un colis du Type A contient des matières radioactives d'une activité maximale A1 s'il s'agit de matières radioactives sous forme spéciale (dont les sources scellées), ou A2 dans le cas contraire. Il est conçu pour satisfaire les prescriptions générales et particulières (dimensions, tenue du colis à la chute, la température, la pression...) dans les conditions normales de transport (i.e. comprend des accidents mineurs).

Colis du Type B : un colis du Type B ne doit pas contenir des quantités d'activité plus grandes que celles qui sont autorisées par l'agrément. Il est conçu pour satisfaire les prescriptions générales et particulières (dimensions, tenue du colis à la chute, la température, la pression...) dans les conditions accidentelles sévères.

A1 et $\mathbf{A 2}$ : sont des valeurs d'activité en $\mathrm{TBq}$ pour chaque radionucléide et listées dans l'ADR.

Programme d'assurance de la qualité pour le transport : ensemble des procédures encadrant les activités liées au transport : conception, fabrication, épreuves, établissement des documents, préparation, envoi, chargement, acheminement, entreposage en transit, déchargement et réception au lieu de destination final, détermination des TI, classement du colis, étiquetage et marquage, contrôles avant expédition, matériels de bord, instructions d'arrimage, documents de bord, surveillance des véhicules, placardage, procédures d'urgence pour garantir la conformité à l'ADR.

Programme de Protection Radiologique relatif au transport (RPP) : ensemble des dispositions systématiques qui régissent le transport pour que les mesures de protection radiologique soient dûment prises en considération : définition de sa portée, rôles de chaque intervenant, évaluation de dose, consignes de sécurité... (ADR 1.7.1.3.2). Porter une attention particulière à la dose reçue par le conducteur du véhicule pendant les transports du gammagraphe.

Expéditeur : celui qui présente un envoi pour le transport et qui est désigné comme étant l'expéditeur dans le document de transport. Avec la disparition de la déclaration permanente d'expédition (voir ci-dessous), l'entreprise de contrôle propriétaire de la source reste l'expéditeur responsable du transport tout en se soumettant aux règles du site industriel en matière de contrôles radiologiques du colis radioactif et de gestion des sources. 


\section{Jusqu'au 31/12/2008 les dispositions suivantes s'appliquaient encore aux gammagraphes}

Il s'agit des dispositions principales suivantes reprises dans l'article 49 de l'arrêté ADR modifié fin 2007 applicables aux transports intérieurs de la France :

a) La clé doit faire l'objet d'une expédition distincte sauf si le transport est effectué par le titulaire de l'autorisation ou un préposé titulaire du CAMARI, la clé peut être conservée, séparément de l'appareil, par le conducteur.

b) L'entreprise doit avoir un système qualité certifié ISO 9001 sauf si les transports sont effectués par une personne titulaire de l'autorisation ou un préposé (titulaire du CAMARI).

c) une déclaration permanente d'expédition valable 1 an peut être établie selon l'annexe D3 de l'arrêté.

d) pas de plaques-étiquettes 7D et un seul extincteur est exigé sur les voitures particulières.

Donc à partir du $1^{\mathrm{er}}$ janvier 2009 ces dispositions particulières ne seront donc plus applicables.

Nota : EDF qualifie les entreprises ses entreprises de contrôles. Dorénavant leur aptitude au transport des matières radioactives sera évaluée lors des qualifications.

\section{Marquage, étiquetage et signalisation}

5.1. Sur le gammagraphe de manière visible, lisible et durable (ADR 5.2.1.7) on doit trouver :

a) Identification de l'expéditeur et/ou du destinataire.

b) Numéro ONU précédé des lettres « UN » : UN2916 (si Type B(U) ou UN2915 (si Type A).

c) Désignation officielle du transport: "Matières radioactives en colis de Type $\mathrm{B}(\mathrm{U})$ » ou « Matières radioactives en colis de Type $\mathrm{A}$ ».

d) Cote du certificat d'agrément (pour les colis du Type B(U)).

e) Indicatif du pays d'origine du modèle de colis (et nom du fabricant pour les colis du Type A).

f) Numéro de série propre à l'emballage conforme au modèle.

g) Mention « TYPE B(U) » ou « TYPE A ».

h) Symbole du trèfle gravé estampé. 
i) Étiquettes de danger 7B ou 7C sur lesquelles sont notées :

- l'indice de Transport (TI),

- l'activité (en Bq),

- le radionucléide contenu (la source).

\subsection{Sur la voiture particulière}

Panneau orange UN 2916 à l'avant et à l'arrière du véhicule.

À partir du 01/01/09 : Placards 7D sur les côtés et à l'arrière.

\subsection{Autre véhicule}

Placards 7D sur les côtés et à l'arrière + panneau orange à l'avant et à l'arrière (ADR 5.3).

\subsection{Le document de transport}

Le document de transport est rédigé selon le modèle $\mathrm{D} 3$ pour les gammagraphes du Type B (selon l'ADR 5.4.1.2.5 pour les colis du Type A).

À partir du 01/01/09 : document de transport à rédiger pour chaque expédition.

\subsection{Signalisation orange (article 28 de l'arrêté ADR)}

Dans le cas de matières radioactives emballées transportées sous utilisation exclusive, lorsque la taille et la construction du véhicule sont telles que les dimensions des panneaux orange peuvent, en application du 5.3.2.2.1 de l'ADR, être ramenées à $300 \mathrm{~mm}$ pour la base, $120 \mathrm{~mm}$ pour la hauteur et $10 \mathrm{~mm}$ pour le liseré noir, seul le numéro ONU est nécessaire et la taille des chiffres prévue au 5.3.2.2.2 peut être réduite à $65 \mathrm{~mm}$ de haut et $10 \mathrm{~mm}$ d'épaisseur.

\section{Contrôles du véhicule}

Sont contrôlés les éléments suivants :

a) Lot de bord (cale, 2 signaux d'avertissement autoporteurs, vêtement fluorescent, lampe de poche,...).

b) Un extincteur poudre $2 \mathrm{~kg}$ si véhicule VP (voiture particulière). En cas de véhicule non- $\mathrm{VP}<3,5$ t et à partir du 01/01/09 pour les véhicules $\mathrm{VP}$, il faut un $2^{\mathrm{e}}$ extincteur poudre de capacité mini $4 \mathrm{~kg}$.

c) Existence du dispositif d'arrimage (état des points d'ancrage et des sangles, ...) dans le véhicule.

d) Moyen de télécommunication (ex : téléphone portable). 


\section{Contrôles au départ et à l'arrivée sur un site du gammagraphe}

\subsection{Les documents}

a) Certificat d'agrément du modèle de colis en cours de validité.

b) Autorisation de détention et d'utilisation de sources sur chantiers extérieurs en cours de validité.

c) Certificat d'agrément d'un modèle de source radioactive sous forme spéciale (Activité source $<10 \mathrm{~A} 1$ ).

d) Attestation de formation du conducteur pour la classe 7 .

e) Certificat qualité de transport (supprimé au 01/01/2009).

f) Le CAMARI du conducteur.

g) Le document de transport (déclaration permanente D3 pour les colis du Type B(U) jusqu'en décembre 2008).

À noter : SI les conducteurs doivent suivre un cours de spécialisation formalisé par un certificat de formation du conducteur classe 7 avec recyclage tous les 5 ans, l'ensemble du personnel pouvant être amené à intervenir dans les opérations de transport doit suivre une formation de sensibilisation aux dangers des rayonnements ionisants.

\subsection{Le colis}

a) Contrôle de l'arrimage dans le véhicule.

b) Contrôle de l'étiquetage, marquage du colis et signalisation du véhicule.

c) Mesures de l'intensité de rayonnement du colis, du véhicule :

- valeur limite au contact du colis : $2 \mathrm{mSv} / \mathrm{h}$,

- valeur limite à $2 \mathrm{~m}$ du véhicule : $0,1 \mathrm{mSv} / \mathrm{h}$,

- frottis de $300 \mathrm{~cm}^{2}$ à l'intérieur du véhicule $<4 \mathrm{~Bq} / \mathrm{cm}^{2}\left(0,4 \mathrm{~Bq} / \mathrm{cm}^{2}\right.$ préconisé).

d) Réaliser des frottis d'une surface de $300 \mathrm{~m}^{2}$ sur l'ensemble de la surface du gammagraphe et sur les accessoires (et collimateur) : $<4 \mathrm{~Bq} / \mathrm{cm}^{2}$.

e) S'assurer de la présence de la source dans le gammagraphe par une mesure de l'intensité de rayonnement contre le projecteur.

\section{Transport du collimateur}

En cas de présence d'un collimateur contenant de l'Uranium appauvri, vérification que celui-ci porte bien un numéro d'identification. Dans le cas contraire, on procède à son identification. 
Veiller à le noter sur le document de transport.

Le transport en UN2911 ou UN2912 est à prohiber si utilisation annexe D3 car le transport en «utilisation exclusive» est obligatoire. Préconiser plutôt un transport en « colis excepté » dans une boîte inactive faite de métal ou d'un autre matériau résistant. Sur la boîte sera inscrit le nUN29O9.

\section{Accident de transport}

Si la sûreté repose d'abord sur le colis et sa capacité à résister aux agressions internes ou externes, à ce principe s'ajoutent d'autres exigences relatives à l'efficacité de l'intervention lors d'un accident et à la conformité de l'ensemble des opérations effectuées dans le cadre de l'exploitation des colis.

\subsection{La sûreté du colis}

Les gammagraphes sont des colis du Type B qui par conception résistent aux accidents, même sévères.

Les limitations réglementaires de l'activité rejetée du contenu et de l'intensité de rayonnement au contact du colis permettent que les situations d'accident soient gérables.

Ces limites sont de telle sorte que les conséquences radiologiques restent acceptables pour le personnel d'intervention dans la majorité des cas d'accident. Ces limites sont :

- une perte accumulée du contenu radioactif ne dépassant $\mathbf{A 2}$ par semaine,

- l'intensité de rayonnement à $1 \mathrm{~m}$ de la surface du colis qui ne dépasse pas $10 \mathrm{mSv} / \mathrm{h}$.

La limitation de l'activité rejetée et de l'intensité de rayonnement rendent donc possible l'intervention sur les colis endommagés sous réserve de précautions adaptées. Ces précautions sont à déterminer au préalable en fonction des résultats de la recherche des niveaux d'irradiation et de contamination éventuelle sur le colis et dans la zone proche du colis.

\subsection{Les interventions doivent être préparées}

Il s'agit de répondre au courrier de l'ASN réf: DGSNR/SD1/001/2005 du 3/01/2005 qui concerne les procédures d'urgence en cas d'accident de transport.

Les consignes générales de sécurité sont disponibles à bord du véhicule. 
La déclaration permanente décrivant l'envoi et attestant sa conformité accompagne le transport (pour un gammagraphe en colis du Type B(U) c'est l'annexe D.3 à l'arrêté $\mathrm{ADR}$ « déclaration permanente de chargement et d'expédition de matières radioactives $»)$.

Le conducteur est équipé de moyens de radiocommunication.

Le conducteur a suivi un cours de spécialisation formalisé par un certificat de formation du conducteur classe 7 avec recyclage tous les 5 ans (sauf pour un transport en colis du Type A).

Le véhicule satisfait aux prescriptions de la réglementation ADR. La société doit désigner un conseiller à la sécurité titulaire d'un certificat de qualification professionnelle.

Le conducteur s'assure notamment :

- que les étiquettes de danger et les signalisations sont bien apposées ;

- que les équipements d'intervention et de protection individuelle prescrits dans les consignes de sécurité se trouvent à bord du véhicule ;

- que les moyens de liaison permettant de contacter, sans délai, les secours ; les Autorités et l'expéditeur se trouvent à bord du véhicule.

\subsection{Les acteurs en cas d'accident}

Le conducteur (s'il est valide)

- Il applique la consigne de sécurité qui lui a été remise et il :

- secourt les éventuels blessés,

- met en place un premier périmètre de sécurité $(10 \mathrm{~m})$,

- prévient : les pompiers (le 112) auxquels il décrit l'accident,

- prévient son entreprise

- tente de maîtriser les éventuels feux de cabine, moteur, pneu, ...

Les pompiers (premiers secours)

- secourent les éventuels blessés,

- de manière réflexe établissent un périmètre de sécurité de $100 \mathrm{~m}$,

- interdisent l'accès des non intervenants à la zone dangereuse,

- tente de maîtriser le feu éventuel

\section{Les pouvoirs publics}

Ils sont responsables de la maîtrise de la situation et de limitation des conséquences de l'accident, en concertation avec :

- la Préfecture

- les cellules de crise nationales (ASN en particulier)

- l'expertise et moyens techniques de l'IRSN et du CEA

- le site EDF le plus proche si besoin. 


\section{Le matériel d'intervention à bord du convoi}

Outre les équipements de sécurité d'usage général exigés par la réglementation $\mathrm{ADR} / \mathrm{RID}$ des équipements spécifiques pour « une première intervention » en cas d'accident sont à prévoir.

\section{Le matériel pour récupérer le colis accidenté}

Conteneur spécifique pour reprendre le colis défectueux.

TABLEAU I

Nature des risques liés aux transports des gammagraphes.

\begin{tabular}{|c|c|c|c|c|c|}
\hline \multirow{3}{*}{$\begin{array}{c}\text { TYPE } \\
\text { DE MATIERE }\end{array}$} & \multirow{3}{*}{$\begin{array}{c}\text { RADIO- } \\
\text { NUCLEIDE }\end{array}$} & \multicolumn{4}{|c|}{ RISQUES RADIOLOGIQUES POSSIBLES } \\
\hline & & \multirow{2}{*}{$\begin{array}{l}\text { EXPOSITION } \\
\text { (Irradiation } \\
\text { externe) }\end{array}$} & \multicolumn{3}{|c|}{ POLLUTION (Contamination) } \\
\hline & & & $\begin{array}{l}\text { Atmosphérique } \\
\text { (Environnement) }\end{array}$ & $\begin{array}{c}\text { Surfacique } \\
\text { (Environnement) }\end{array}$ & $\begin{array}{l}\text { Corporelle } \\
\text { (Personnes) }\end{array}$ \\
\hline $\begin{array}{l}\text { Source scellée utilisée } \\
\text { à des fins industrielles } \\
\text { (gammagraphe, } \\
\text { irradiateur) }\end{array}$ & $\begin{array}{l}\text { Cobalt } 60 \\
\text { Iridium } 192 \\
\text { Césium } 137 \\
\text { Sélénium } 75\end{array}$ & oui & non (1) & non (1) & non $(1)$ \\
\hline
\end{tabular}

(1) sauf si l'enveloppe de confinement de la source scellée est détruite 